\title{
The Combination of Ultrasound-Guided Pectoral Nerves II and Para- sternal Blocks as a Valid Alternative for Anaesthesia in Breast Surgery: An Observational Study
} Paolo Scimia, $\mathrm{MD}^{1}$, Erika Basso Ricci, $\mathrm{MD}^{1}$, Fabiola Harizaj, $\mathrm{MD}^{1}$, Alessandra Cocconi, $\mathrm{MD}^{2}$, Pierfran-
cesco Fusco, $\mathrm{MD}^{3}$, and Giorgio Danelli, MD

Background: Breast cancer surgery is often associated with severe postoperative pain that may compromise systemic homeostasis, which increases perioperative morbidity, the length of stay in the hospital, and costs. Scientific evidence has also shown that an inadequate analgesia could promote the risk of persistent pain development after breast surgery. Objective: Recent literature suggested that the pectoral nerves II (PECS II) block may represent a valid alternative to general anesthesia (GA) and conventional, regional techniques for analgesia in breast surgery. This technique may provide complete anesthesia of the lateral part of the thorax but cannot block, by itself, the anterior cutaneous branches of the intercostal nerves. The combination of a parasternal block (PSB) and a PECS II block has been performed as a single anesthetic technique.

Study Design: This is an observational, monocenter, prospective, and cohort study. We obtained the approval of our scientific ethic committee and clinical trials registration.

Setting: This study enrolled patients undergoing an elective breast surgery. In particular, we enrolled patients who were scheduled for a mastectomy or quadrantectomy of the medial part of the breast.

Methods: We recruited 40 patients who were scheduled for breast surgery. A PECS II block was performed with an injection of ropivacaine $0.5 \%$ $20 \mathrm{~mL}+10 \mathrm{~mL}$. Then, a PSB was performed by 2 separate injections of $3 \mathrm{~mL}$ of $0.5 \%$ ropivacaine, for each one, at the level of the second and fourth intercostal space. All of the patients received intraoperative sedation and multimodal analgesia. During the intraoperative period, the accessory need of a local anesthetic infiltration, conversion to GA, and the total amount of propofol required to maintain good comfort of the patients were recorded. In the first 24 postoperative hours, every 6 hours, postoperative pain was assessed by an investigator using a numerical rating scale (NRS). The consumption of analgesic and antiemetic drugs and the incidence of postoperative nausea and vomiting (PONV) were also recorded.

Results: Our observational analysis yielded 40 patients in a period of 6 months. The population was subdivided into 2 groups: a mastectomy group or a quadrantectomy group. All of the population reported their pain scores at rest ( $r N R S<3$ ) and during activity (iNRS < 5) in the postoperative period. None of the patients required GA. Six patients $(27.3 \%)$ in the mastectomy group required a supplemental anesthetic infiltration. Eleven (27.5\%) patients required a rescue analgesic drug: $9(40.9 \%)$ in the mastectomy group and $2(11.1 \%)$ in the quadrantectomy group. Two patients reported events of PONV, one for each group $(4.54 \%$ for the mastectomy group and $5.55 \%$ for the quadrantectomy group). No complications occurred.

Conclusion: This study indicates the safety and feasibility of the novel ultrasound-guided thoracic wall blocks during inpatient and outpatient breast surgery for the management of intraoperative anesthesia and postoperative analgesia.

Limitations: This is an observational study; a randomized control trial is mandatory to confirm the results.

Key words: Breast cancer surgery, pectoralis nerve block, parasternal block, ultrasound-guided anesthesia, regional anesthesia, pain control

From : ${ }^{1}$ Department of Anesthesia and Perioperative Medicine, Hospital of Cremona, Cremona, Italy; ${ }^{2}$ Breast Surgery Unit, Hospital of Cremona, Cremona, Italy; ${ }^{3}$ Department of Anesthesia and Intensive Care Unit, S. Salvatore Hospital, L'Aquila, Italy; ${ }^{4}$ Department of Anesthesia and Intensive Care Unit, IRCCS di Reggio Emilia, Reggio Emilia, Italy

Author for correspondence: Erika Basso Ricci, MD

Address: Department of Anesthesia and Perioperative Medicine, A.S.S.T. Cremona, Viale Concordia 1, Cremona, Italy

E-mail: erika.bassoricci@gmail.com 
Breast cancer surgery is often associated with severe postoperative pain that may compromise systemic homeostasis, which increases perioperative morbidity, the length of stay in the hospital, and costs. Scientific evidence also shows that an inadequate analgesia could promote the risk of persistent pain development after breast surgery (1).

Recent literature suggested that the ultrasoundguided pectoral nerves II (PECS II) block may represent a valid alternative to general anesthesia (GA) and conventional, regional techniques for analgesia in breast surgery $(2,3)$.

A PECS II block, described by Blanco et al (4), may provide complete anesthesia of the lateral part of the thorax in breast surgery (5).

However, this technique cannot block, by itself, the anterior cutaneous branches of the intercostal nerves that pass near the sternum that provide the innervation of the integument of the front of the thorax and the medial aspect of the mammary gland. Medial quadrantectomy or radical mastectomy requires a reliable block of the anterior rami of the intercostal nerve T2-T6.

Tighe et al (5) suggested that the serratus plane block (SPB) does not provide anesthesia for the medial part of the breast. This hypothesis led Fusco et al (6) to combine a SPB with a parasternal block (PSB) to guarantee complete anesthesia of the entire area of the breast. In accordance to this suggestion, we hypothesized that the combination of a PECS II block with a PSB could provide a reliable block of the anterior and lateral rami of the intercostal nerves, in order to ensure complete anaesthesia for surgery, involving both the lateral and medial quadrants of the breast.

In our observational study, the combination of a PSB and PECS II block has been performed as a single anesthetic technique in patients scheduled to undergo a radical mastectomy or medial quadrantectomy, with or without axillary dissection.

The patients were evaluated during the intraoperative period and for 24 hours postoperatively. Any need to change the anesthetic management was recorded. The secondary end-points were the control of postoperative pain, the incidence of postoperative nausea and vomiting (PONV), the consumption of analgesic and antiemetic drugs, and the analysis of block-related complications.

\section{METHODS}

\section{Study Design, Setting, and Recruitment}

This is an observational, mono-center, longitudinal, and cohort study that took place at the Hospital of Cremona. We obtained the approval of our scientific ethic committee and clinical trials registration. After the research ethic committee gave written informed consent, we enrolled 40 patients, who were at least 18 years old, with the American Society of Anesthesiologists (ASA) physical status of I-II-III, who were scheduled for elective breast surgery between July 2016 and November 2016. The exclusion criteria were loco-regional anesthesia contraindication, toxic abuse history, neuropathic disease, and refusal of the patient. During the preoperative visit, demographic data were recorded and the numerical rating scale (NRS; 0-10, 0 = no pain, 10 = worst pain) was explained to the patients.

After informed consent and preoperative sedation with midazolam one milligram and fentanyl $50 \mathrm{mcg}$, a PECS II block was performed under ultrasound guidance. The patients were placed in the supine position with the ipsilateral upper limb in abduction at 90 degrees. After skin sterilization, a $15 \mathrm{MHz}$ linear probe was first placed on the infraclavicular region at the level of the first rib, where the pectoralis muscles and subclavian vessels were identified. Then, the transducer was moved laterally toward the axilla, where the serratus muscle was identified at the level of the fourth rib. A22 gauge needle (SonoTap, Pajunk ${ }^{\circledR}$, Geisingen, Germany) was inserted with an in-plane approach from the cranial to caudal direction, confirming the correct needle-tip position by hydrolocalization with normal saline solution. Ropivacaine $0.5 \% 20 \mathrm{~mL}+10$ $\mathrm{mL}$ was injected into the fascial planes between the pectoralis minor and serratus muscles and between the pectoralis muscles, respectively. Then, an ipsilateral PSB was performed under ultrasound guidance by 2 separate injections of $3 \mathrm{~mL}$ of $0.5 \%$ ropivacaine, for each one, at the level of the second and fourth intercostal space, between the major pectoral and intercostal muscles, underneath the external intercostal membrane. A $15 \mathrm{MHz}$ linear probe was positioned at the level of the second rib along the parasternal line, where the external intercostal membrane between the major pectoral and intercostal muscles was identified. A 22 gauge needle was inserted with an in-plane approach from the cranial to caudal direction 
PECS and Parasternal Blocks in Breast Cancer Surgery

and, after an injection of $1-2 \mathrm{~mL}$ of saline solution to open this space, $3 \mathrm{~mL}$ of $0.5 \%$ ropivacaine solution was injected. The same procedures were performed at the fourth rib. Because the anterior branches of the intercostal nerve penetrate through these 2 muscles to innervate the internal mammary area, we hypothesized that an injection of local anesthetic into the interfascial compartment could block the same nerves (7). Our diffusion technique was performed by injecting a volume of $3 \mathrm{~mL}$ of local anesthetic in correspondence to the 2 different intercostal spaces, in order to promote the spread of the anaesthetic solution and achieve a sensory block extended to the proximal dermatomes of the intercostal nerve near to the site of injection (7).

All of the patients received intraoperative sedation with an intravenously propofol infusion (TIVA-TCI 1-2 $\mathrm{mcg} / \mathrm{mL}$ ), monitoring the bispectral index (BIS) with a target of 50-60 values. Supplemental oxygen (4-6 $\mathrm{l} / \mathrm{min}, 50 \%$ of the fraction of inspired oxygen) was administered by a venturi mask under an end-tidal $\mathrm{CO}_{2}$ control $\left(\mathrm{EtCO}_{2}\right)$ to ensure an oxygen saturation $\left(\mathrm{SaO}_{2}\right)$ more than $90 \%$; the patient's heart rate, electrocardiogram (ECG), arterial blood pressure, and $\mathrm{SaO}_{2}$ were monitored during the surgery.

During the intraoperative period, the accessory need of a local anesthetic infiltration, conversion to $\mathrm{GA}$, and the total amount of propofol required to maintain good comfort of the patients were recorded. In the first 24 postoperative hours, every 6 hours, the patients' pain was assessed by an investigator using a NRS. The consumption of analgesic and antiemetic drugs and PONV were also recorded.

The scheduled postoperative analgesic was acetaminophen one gram every 8 hours, with a rescue dose of ketorolac $30 \mathrm{mg}$.

To reduce the source of bias, all of the patients received loco-regional anesthesia and sedation by 3 skilled anesthesiologists, and surgery was performed by a single surgeon.

All of the patients who were treated by the authors in a period of 6 months were enrolled in this study.

Continuous data are presented as mean $\pm S D$, and binary data are presented as the percentages of patients. We included a convenience sample of patients with no previous sample size calculation, wherefore the sample size was determined by the number of patients eligible within the inclusion pe- riod. Descriptive data were stored in Microsoft Excel 2010 (Microsoft Corporation, Redmond, WA), and for statistical analyses we used SPSS Version 24.0 (IBM Corporation, Armonk, NY).

\section{RESULTS}

Our observational analysis yielded 40 patients who underwent elective breast surgery in a period of 6 months. Fifty-seven patients met the eligibility criteria, but only 40 of them received anesthesia by the authors.

All of the patients received the combination of 2 ultrasound-guided chest wall blocks. Table 1 shows the demographical data of the patients enrolled in the analysis. The population was subdivided by the type of breast surgery they were undergoing: mastectomy with lymphadenectomy (mastectomy group $=22$ patients) or medial quadrantectomy with sentinel lymphonode biopsy (SLNB) (quadrantectomy group $=18$ patients).

We recorded the NRS values (at rest $=$ rNRS and during activity $=$ iNRS) registered at the scheduled times (Table 2). In all of the population, the observed rNRS values reflected perfect postoperative pain control (rNRS values $<3$ and iNRS $<5$ ).

None of the patients required conversion to GA. The total amount of propofol required to maintain good comfort of the patients was $2.64 \pm 1.14 \mathrm{ng} /$ $\mathrm{kg} / \mathrm{h}$ for the entire population of the study. The mastectomy group patients required a mean of $3 \pm 1.62$ $\eta \mathrm{g} / \mathrm{kg} / \mathrm{h}$ of propofol for intraoperative sedation, and the quadrantectomy group received a mean of $2.3 \pm$ $0.67 \mathrm{ng} / \mathrm{kg} / \mathrm{h}$ of propofol.

Six patients $(27.3 \%)$ of the mastectomy group received an additional local anesthetic infiltration from the surgeon. Eleven (27.5\%) patients required a rescue analgesic drug (ketorolac $30 \mathrm{mg}$ ) in the postoperative period: $9(40.9 \%)$ in the mastectomy group and $2(11.1 \%)$ in the quadrantectomy group. All of

Table 1. Demographic data of the included patients.

\begin{tabular}{|l|l|l|l|}
\hline & $\begin{array}{l}\text { Age, yrs } \\
(\text { mean } \pm \text { SD) }\end{array}$ & $\begin{array}{l}\text { Weight, kg } \\
(\text { mean } \pm \text { SD) }\end{array}$ & $\begin{array}{l}\text { Height, cm } \\
(\text { mean } \pm \text { SD) }\end{array}$ \\
\hline Population (n= 40) & $70.15 \pm 16.50$ & $70.06 \pm 16.50$ & $163.29 \pm 7.26$ \\
\hline $\begin{array}{l}\text { Mastectomy } \\
(\mathrm{n}=22)\end{array}$ & $75.22 \pm 13.97$ & $74.31 \pm 19.19$ & $162.56 \pm 8.08$ \\
\hline $\begin{array}{l}\text { Quadrantectomy + } \\
\text { SLNB (n=18) }\end{array}$ & $64.07 \pm 17.68$ & $65.53 \pm 12.09$ & $164.06 \pm 6.45$ \\
\hline
\end{tabular}


Table 2. The NRS scores in the postoperative period (T0: time at operative room discharge; T6: time at 6 postoperative hours; T12: time at 12 postoperative hours; T24: time at 24 postoperative hours; rNRS: NRS score at rest; iNRS: NRS score during activity).

\begin{tabular}{|c|c|c|c|c|c|c|c|c|}
\hline & $\begin{array}{c}\text { rNRS T0 } \\
(\text { mean } \pm \text { SD) }\end{array}$ & $\begin{array}{c}\text { iNRS T0 } \\
\text { (mean } \pm \text { SD) }\end{array}$ & $\begin{array}{c}\text { rNRS T6 } \\
(\text { mean } \pm \text { SD) }\end{array}$ & $\begin{array}{c}\text { iNRS T6 } \\
\text { (mean } \pm \text { SD) }\end{array}$ & $\begin{array}{c}\text { rNRS T12 } \\
(\text { mean } \pm \text { SD) }\end{array}$ & $\begin{array}{c}\text { iNRS T12 } \\
(\text { mean } \pm \text { SD) }\end{array}$ & $\begin{array}{c}\text { rNRS T24 } \\
(\text { mean } \pm \text { SD) }\end{array}$ & $\begin{array}{c}\text { iNRS T24 } \\
(\text { mean } \pm \text { SD) }\end{array}$ \\
\hline $\begin{array}{l}\text { Population } \\
(\mathrm{n}=40)\end{array}$ & $0.94 \pm 1.43$ & $3.53 \pm 1.44$ & $1.78 \pm 1.50$ & $4.52 \pm 1.36$ & $1.00 \pm 1.24$ & $3.43 \pm 1.33$ & $0.17 \pm 0.58$ & $0.43 \pm 0.93$ \\
\hline $\begin{array}{l}\text { Mastectomy } \\
(\mathrm{n}=22)\end{array}$ & $1.44 \pm 1.69$ & $3.87 \pm 1.74$ & $1.77 \pm 1.69$ & $4.36 \pm 1.50$ & $1.11 \pm 1.36$ & $4.00 \pm 1.63$ & $0.44 \pm 0.88$ & $1.14 \pm 1.35$ \\
\hline $\begin{array}{l}\text { Quadrantectomy } \\
+ \text { SLNB }(\mathrm{n}=18)\end{array}$ & $0.37 \pm 0.81$ & $3.18 \pm 0.98$ & $1.79 \pm .37$ & $4.64 \pm 1.28$ & $0.93 \pm 1.21$ & $3.14 \pm 1.10$ & & $0.07 \pm 0.26$ \\
\hline
\end{tabular}

Table 3. The incidence of PONV events, required GA, rescue analgesic drugs, and local anesthetic infiltration from the surgeon.

\begin{tabular}{|l|l|l|l|l|}
\hline & $\begin{array}{l}\text { Incidence } \\
\text { of PONV } \\
(\mathrm{n} ; \%)\end{array}$ & $\begin{array}{l}\text { Conversion } \\
\text { to GA } \\
(\mathrm{n} ; \%)\end{array}$ & $\begin{array}{l}\text { Required Rescue } \\
\text { Analgesic Drugs } \\
\text { (n; \%) }\end{array}$ & $\begin{array}{l}\text { Local } \\
\text { Anesthetic } \\
\text { Infiltration } \\
\text { (n; \%) }\end{array}$ \\
\hline $\begin{array}{l}\text { Population } \\
(\mathrm{n}=40)\end{array}$ & $2 ; 5$ & $0 ; 0$ & $11 ; 27.5$ & $6 ; 15$ \\
\hline $\begin{array}{l}\text { Mastectomy } \\
(\mathrm{n}=22)\end{array}$ & $1 ; 4.54$ & $0 ; 0$ & $9 ; 40.90$ & $6 ; 27.3$ \\
\hline $\begin{array}{l}\text { Quadrantectomy } \\
+ \text { SLNB (n=18) }\end{array}$ & $1 ; 5.55$ & $0 ; 0$ & $2 ; 11.11$ & $0 ; 0$ \\
\hline
\end{tabular}

the patients in the mastectomy group received paracetamol one gram every 6 hours for the first 24 hours. A single dose of paracetamol was administered to the patients of the quadrantectomy group. Two patients reported events of PONV (defined as a necessity to assume postoperative antiemetics drugs), one for each group (4.54\% for the mastectomy group, $5.55 \%$ for the quadrantectomy group) (Table 3). No complications occurred during and after the execution of the chest wall blocks.

\section{DISCUSSION}

This study describes the combination of PSB and PECS II blocks as a novel anaesthetic and analgesic technique in breast surgery.

Complete anesthesia from T2 to T7 dermatomes was observed, and the patients were determined to be ready for surgery. No incidents of pneumothorax, chest wall lesions, infections, or side effects from the local anesthetics were recorded. A reliable anesthesia of the mammary and axillary regions was obtained, with a good hemodynamic stability and without discomfort for the patient and surgeon. No supportive or pharmacological treatments were initiated to correct the values of the principal vital parameters of the patient.

In the first 24 hours after surgery, the patients reported good quality analgesia with a NRS score < 3. No PONV and other complications were recorded. The patients remained comfortable throughout the postoperative period with optimal pain relief and were discharged on the second postoperative day with oral analgesics and follow-up instructions.

After hospital discharge, the patients were followed-up for pain and drug requests within 3 months via self-reporting, and no postoperative persisted pain syndrome has been reported.

This observational study identifies the safety and feasibility of the novel ultrasound-guided thoracic wall blocks during inpatient and outpatient breast surgery for the management of intraoperative anesthesia and postoperative analgesia. Although in literature there are few reports, scientific evidence suggests that these techniques could significantly improve the surgical outcome of the patients undergoing breast cancer surgery (8).

To the authors' knowledge, this is the first description of a case series, in which a PSB in combination with a PECS II block was performed as a single anaesthetic technique in a radical mastectomy or a medial quadrantectomy, with or without axillary dissection. A randomized controlled trial is mandatory to confirm our hypothesis.

Although, as suggested by Karmakar et al (9), thoracic epidural analgesia and paravertebral block represent the gold standard in breast surgery, this novel approach could be 
considered a viable alternative to GA and conventional, regional techniques, especially when the other options could be not recommended or if the patient is high risk. Our experience, although very limited, is encouraging. Therefore, further studies are needed to demonstrate the validity of this approach as an anesthetic standardized method for breast surgery. Another interesting field of research could be focused on verifying if these techniques may play a role in the prevention of persistent postoperative pain after breast cancer surgery.

\section{Funding}

The authors do not have any sources of funding to report.

\section{REFERENCES}

1. Andreae, $\mathrm{MH}$, Andreae, DA. Regional anaesthesia to prevent chronic pain after surgery: A Cochrane systematic review and meta-analysis. Br J Anaesth 2013; 111:711-720.

2. Blanco R. The 'pecs block': A novel technique for providing analgesia after breast surgery. Anaesthesia 2011; 66:847-848.

3. Huercio I, Abad-Gurumeta A, Gilsanz F. Regional blocks for breast surgery: Is it enough? Minerva Anestesiol 2016; 82:369370.

4. Blanco R, Fajardo M, Parras Maldonado T. Ultrasound description of pecs II (modied pecs I): A novel approach to breast surgery. Rev Esp Anestesiol Reanim 2012; 59:470-475.

5. Tighe SQ, Karmakar MK. Serratus plane block: Do we need to

learn another technique for thoracic wall blockade? Anaesthesia 2013; 68:1103-1106.

6. Fusco P, Scimia P, Petrucci E, Di Carlo S, Marinangeli F. The ultrasound-guided parasternal block: A novel approach for anesthesia and analgesia in breast cancer surgery. Minerva Anestesiol 2017; 83:221-222.

7. Moore DC. Intercostal nerve block: Spread of india ink injected to the ribs costal groove. Br J Anaesth 1981; 53:325-329.

8. Marret E, Vigneau A, Salengro A, Noirot A, Bonnet F. Effectiveness of analgesic techniques after breast surgery: A meta-analysis. Ann Fr Anesth Reanim 2006; 25:947-954.

9. Karmakar MK. Thoracic paravertebral block. Anesthesiology 2001; 95:771-780. 